
\title{
$\checkmark$ Research Square \\ Effect of Lentivirus-mediated GDF5 Transfection on Differentiation of Rabbit Nucleus Pulposus Mesenchymal Stem Cells
}

kun zhu

Bengbu Medical College https://orcid.org/0000-0001-8359-6935

Rui Zhao

Bengbu Medical College

Yuchen Ye

Affiliated Hospital of Bengbu Medical College

Gang Xu

Affiliated Hospital of Bengbu Medical College

Changchun Zhang ( $\sim$ zccanhui@sina.com )

Affiliated Hospital of Bengbu Medical College

Research

Keywords: Nucleus pulposus mesenchymal stem cells, lentivirus, transfection, GDF5, disc degeneration

Posted Date: July 7th, 2020

DOI: https://doi.org/10.21203/rs.3.rs-40034/v1

License: (a) (i) This work is licensed under a Creative Commons Attribution 4.0 International License. Read Full License 


\section{Abstract}

Background: Disc degenerative disease is a common senile degenerative disease, which seriously affects the quality of life of patients. The purpose of this study is to observe the biological and cytological characteristics of rabbit nucleus pulposus mesenchymal stem cells (NPMSCs), and to determine the effect of growth differentiation factor 5(GDF5) on the differentiation of rabbit NPMSCs by lentivirus transfection.

Methods: In vitro culture model of rabbit NPMSCs was established and NPMSCs cells were identified by flow cytometry (FCM) and quantitative real-time PCR(qRT-PCR). Then NPMSCs were divided into three groups: lentiviral vector carrying GDF5 was used to transfect NPMSCs, to determine the transfection rate, which was recorded as transfection group, and the NPMSCs transfected with ordinary lentiviral vector was recorded as control group, NPMSCs without processing was recorded as normal group. FCM, qRTPCR and Western Blot(WB) were used to detected the change of NPMSCs.

Results: The transfected NPMSCs by GDF5 became longer and narrower, and the cell density decreased,and the positive rate of GDF5 in the transfected group was significantly higher than that in the other two groups $(\mathrm{P}<0.05)$. The mRNA expression of KRT8, KRT18, KRT19 in the transfected group was significantly higher than the other two groups $(P<0.05)$,the result of WB were the same to qRT-PCR.

Conclusions: GDF5 can induce the differentiation of NPMSCs and repair degenerative intervertebral discs. Lentiviral vector carrying GDF5 can be integrated into the chromosome genome of NPMSCs and promote differentiation of NPMSCs into nucleus pulposus cells(NPCs).

\section{Background}

Disc degenerative disease is a common senile degenerative disease, which seriously affects the quality of life of patients. Apoptosis of nucleus pulposus cells and the decrease of cell viability are the key factors leading to disc degeneration, which is often accompanied by a decrease in extracellular matrix synthesis such as type II collagen and glycoprotein [1, 2]. The traditional treatment methods are surgical intervention and conservative treatment, the former is traumatic, the latter is not effective, and the two methods can not fundamentally restore the biological characteristics of the intervertebral disc. If the number of nucleus pulposus cells can be supplemented by artificial intervention, it is possible to fundamentally reverse the process of disc degeneration. It is reported that there is a type of stem cell in the nucleus pulposus with a strong ability to proliferate and differentiate, which is the nucleus pulposus mesenchymal stem cell (NPMSCs) [3, 4]. In this study, GDF5 gene was used to intervene NPMSCs of rabbits in vitro, and the effect of GDF5 gene on differentiation of medullary nucleus mesenchymal stem cells was observed.

\section{Materials And Methods}


Adult healthy rabbits (purchased from experimental animal feeding and management center of Bengbu medical college).

\section{Main materials and instruments}

\section{Cells}

rabbit NPMSCs, isolated and cultured in our laboratory.

\section{Instruments}

ultra-clean working table (Bengbu HVAC Purification Equipment co., LTD.), cell culture box (American Saimofei Technology co., LTD.), micro-centrifuge (Hangzhou Aosheng Instrument co., LTD.), PCR instrument (Hangzhou Lattice Scientific Instrument Co., LTD.), flow cytometry (Becton Dickinson, USA).

Reagents: DMEM (Gibco, C11995500CP), RPMI 1640 (Gibco, C11875500BT) fetal bovine serum (Bio IND, 04-002-1 a), Antibiotic-Antimycotic (Lifetechnologies, 15240-112); PBS, pH7.4 (Gibco, 10010-023); Trypsin-edta (0.05\%) (Lifetechnologies, 25300-054); Bovine serum albumin (Lifetechnologies, 15561012); Lipofectamine ${ }^{\circledR} 2000$ Transfection Reagent: Lifetechnologies, 11668-019; Opti-mem ® I Reduced Serum Medium: Lifetechnologies, 31985-062; Western blot and IP cell lysis solution; Predye protein Marker (Fermentas, Lithuania); Poluvinylidene membrane (Millipore, USA).

\section{Antibody}

CD90antibody (ABCAM, ab225); CD105antibody (ABCAM, ab221675); CD34antibody (Bioss, bs-0646R); CD45antibody (Bioss, bs-0522R); Fitc-labeled sheep anti-mouse IgG (Bioss, bs-0296 g-fitc); Fitc-labeled sheep anti-rabbit IgG (Bioss, bs-0295 g-fitc); GDF5 wereantibody (ABCAM, ab93855); Anti-actin antibody (Sigma, USA).

Kit

Ultrapure RNA Kit RNA extraction Kit, kangwei century (CW0581S); SuperRT cDNA Synthesis Kit, kangwei century (CW0741S); UltraSYBR Mixture (High ROX), kangwei century (CW2602M); BCA protein quantitative kit (Shanghai biyuntian institute of biotechnology).

\section{Cell isolation culture and identification}

Acquisition of NPMSCs The rabbit nucleus pulposus tissues were obtained, digested by collagenase, centrifuged and resuspended, nucleus pulposus cells were obtained. Then the nucleus pulposus cells (NPCs) suspension was centrifuged and the supernatant fluid was removed and the complete medium of mesenchymal stem cells were added, and finally suspension was performed to obtain NPMSCs. When the cell fusion reached $80 \%-90 \%$, the cell could be digested by the trypsin and re-inoculated in the culture 
bottle for the subculture of NPMSCs. The morphological and biological characteristics of cells were observed with inverted microscope.

The surface immunophenotype of NPMSCs in rabbits was identified by flow cytometry (FCM). The third generation NPMSCs cells were digested with trypsin, centrifuged, and then the precipitated cells were collected, washed by PBS, and diluted to cell suspension. The anti-rabbit CD90, CD105, CD34, CD45 were added, the suspension was incubated at room temperature, washed by PBS, and incubated with FITClabeled sheep anti-mouse/sheep anti-rabbit IgG second antibody away from light, washed by PBS. After mixing, the mixture was detected by FCM (six times).

Quantitative Real-time PCR (qRT-PCR) collects samples, extracts RNA, detects the mRNA level of the target gene by qRT-PCR. RNA from the NPMSCs was extracted, and then cDNA was synthesized. Then qRT-PCR was performed, included internal reference GAPDH gene, and each gene made three parallel holes per sample. After adding each component to the PCR tube, carefully seal the plate membrane, mix evenly, and simply centrifuge the solution to the bottom of the tube. PCR amplification conditions were based on the instructions of the qRT-PCR kit. According to the instructions of the instrument, the PCR experiment was carried out (six times). The data was collected and the results were analyzed.

\section{Cell transfection and observation}

NPMSCs were divided into three groups: lentiviral vector carrying GDF5 gene was used to transfect NPMSCs, to determine the transfection rate, which was recorded as transfection group, and the NPMSCs, transfected with ordinary lentiviral vector was recorded as control group, without processing, NPMSCs was recorded as normal group. Transfection methods: the lentivirus plasmids were diluted to $25 \mathrm{~L}$ of optimem ${ }^{\circledR}$ I Reduced Serum Medium, and $0.5 \mathrm{~L}$ of Lipofectamin2000 was diluted to $25 \mathrm{~L}$ of opti-mem $\AA$ I Reduced Serum Medium, respectively. Then the two solutions were mixed gently and left to rest, finally the mixture were added to three groups of cell culture Wells for culturing.

FCM identified the gene transfection rate, the three groups of cells were digested with trypsin and centrifuged, and the precipitated cells were collected, and diluted cell suspension after washed by PBS and added anti-rabbit GDF5 respectively, incubated. The cells were incubated with FITC-labeled sheep anti-rabbit IgG second antibody and washed by PBS. After mixing, the positive rate of GDF5 in the three groups was detected by FCM (six times in each of the three groups).

qRT-PCR was used to detect the mRNA expression of keratin 8, 18, 19(KRT8, KRT18, KRT19) in three groups. Cell samples were collected, RNA was extracted and then cDNA synthesis was carried out. Finally, the sample were detected by FQ PCR (six times in each of the three groups). The method steps were the same as 1.13 , and the procedures were not described in detail.

The protein expression levels of KRT8, KRT18 and KRT19 in the three groups were detected by Western Blot (WB). Steps include: the extraction of total protein of three groups of cells, quantitative of protein, protein electrophoresis, transfer film, sealing and incubation, PVDF membrane chemiluminescence, developing and fixing, one slice for each case (six times in each of the three groups), the final image 
analysis: the optical density of the developing strip was analyzed and scanned by Image $\mathrm{J}$, and the ratio of the optical density of the target band to the corresponding value of $\beta$-actin was the relative expression.

\section{Statistical methods}

The data were expressed as mean \pm standard deviation, One-way anova was used to compare the three groups, and independent sample t test was used for pairwise comparison. Test level $\mathrm{P}=0.05$.

\section{Results}

\section{Culture and identification of NPMSCs}

Most of the NPMSCs screened by the complete culture medium of mesenchymal stem cells were single after digestion (Fig. 1A), some of the cells were adherent to the wall after primary culture for $4 \times 6$ days, and the morphology of the cells was spindle-shaped or polygonal (Fig. 1B). After 12 days, cell colony formation was observed, and 3-4 weeks later the cells fusion rate reached to $90 \%$. After passage, the cells proliferation increased obviously and the following passage takes only about one week, and the spindle cells began to increase. By the third generation, most of the cells were fusiform (Fig. 1C).

FCM showed that the surface immunophenotype CD34, CD45 were negative (Fig. 2B), the surface immunophenotype CD90, CD105 were positive in the detected cells (Fig. 2C). The expression rate of CD molecule of surface immunophenotype of NPMSCs is shown in Fig. 2D.

qRT-PCR results: NPMSCs could express stem cell gene SOX2, Nanog (Fig. 1D).

\section{Detection of GDF5 gene transfected NPM}

\section{SCs}

The transfected NPMSCs became longer and narrower, and the cell density decreased (Fig. 4A).

FCM cell transfection rate: the GDF5 positive rate (\%) of the transfected group was significantly higher than that of the normal group and the control virus group $(P<0.001)$ (Fig. 3D), and the GDF5 expression positive rate (\%) of the three groups (Figs. 3A, 3B, 4C).

The results of qRT-PCR in the three groups showed that the mRNA expression of KRT8, KRT18, KRT19 in the transfected group was significantly higher than that in the other two groups, and the difference was statistically significant $(P<0.05)$ (Fig. 4B).

WB results: the protein expression levels of KRT8, KRT18 and KRT19 in the transfection group were significantly increased compared with the other two groups $(P<0.001)$ (Fig. 4C, 4D).

\section{Discussion}


Lumbar degenerative disease caused by lumbar disc degeneration has seriously affected the life of patients and has become a serious social problem, but both surgery and conservative treatment did not fundamentally reverse disc degeneration $[5,6]$. However, with the development of molecular mechanisms and genetic engineering of disc degeneration, it is possible to treat or reverse disc degeneration at the gene level.

Mesenchymal stem cells (MSCs) are pluripotent stem cells originated from the mesoderm, which can differentiate into bone, cartilage, fat, muscle, ligament, tendon and other tissues [7, 8]. In 2006, the international association for cell therapy proposed a common standard for the definition of mesenchymal stem cells [9]: A. in vitro culture, such cells can grow adherently to the wall; B. Some specific antigens (markers) are expressed on the cell surface; $C$. The ability to differentiate into adipocytes, osteoblasts and chondrocytes. Several studies have shown that there is a kind of cell in the intervertebral disc tissue, and this kind of cell has the ability to differentiate into osteoblasts, chondroblasts and lipid cells. Meanwhile, this kind of cell also characteristically expresses the surface protein molecules of mesenchymal stem cells, which can complete the three-line induced differentiation. Therefore, such cells are named as nucleus pulposus mesenchymal stem cells (NPMSCs) [10-12].

Li XC et al successfully isolated human NPMSCs by fluorescence activated cell sorting and which expressed tyrosine kinase receptor 2 (Tie-2) and ganglioside 2 (GD-2). The cells had significant proliferation and differentiation potential and could differentiate into osteoblasts, adipocytes, and chondrocytes [13]. Erwin WM et al proved that NPMSCs has a powerful ability to divide and proliferate by animal experiments, and played an important role in IVD repair, nerve repair and other renewable medicine [14]. Consequently, NPMSCs is the precursor of NPCs, which exists in normal and degenerative IVD tissues and has the potential to proliferate and differentiate into NPCs. NPMSCs are the precursor cells of nucleus pulposus cells, which exist in normal and degenerated nucleus pulposus tissues and have a strong potential to proliferate and differentiate into nucleus pulposus cells, and can be used in regenerative and repair medicine $[3,4]$.

Lin et al. cultured NPMSCs of rats in vitro and induced differentiation, and found that NPMSCs could express stem cell genes, such as SOX2, Oct4 and Nanog [15]. Hui Zhang et al. compared rat NPMSCs and bone marrow mesenchymal stem cells by in vitro culture, and found that both can differentiate into bone, cartilage and fat, and can express stem cell genes Nanog, oct-4 and sox-2, with no obvious difference [16]. In this study, FCM was used to detect surface immunophenotype CD molecular of NPMSCs. The immunophenotypes CD90 and CD105 expressed positive, and the immunophenotypes CD34 and CD45expressed negative. qRT-PCR was used to detect positive expressions of SOX-2 and Nanog, NPMSCs could express stem cell gene SOX2, Nanog.

Some scholars have found that human notochord cells gradually reduce with the growth of age and disc degeneration, this notochord cells are NPMSCs [17]. Rodrigues-pinto $\mathrm{R}$ et al. found that keratin 8, 18, 19 (KRT8, KRT18, KRT19) are specific markers of human notochord cells, and are expressed in all stages of notochord cells [18]. Minogue et al. detected the mRNA levels of KRT8, KRT18 and KRT19 in bovine 
nucleus pulposus cells and notochord cells by rt-pcr, and found that both expressed KRT8, KRT18 and KRT19 genes, and notochord cells expressed slightly more than nucleus pulposus cells [19]. Meanwhile, some scholars have found that nucleus pulposus cells can also express KRT8, KRT18, KRT19 and other gene phenotypes, and the expression level of nucleus pulposus cells is higher than that of articular chondrocytes and ring fibroblasts [20-22].

GDF5, also known as BMP14, is a member of the bone morphogenetic protein (BMP) family. BMP was originally thought to be a component of the mineralized bone matrix, and when a fracture or ectopic ossification occurred in the body, it can induce the formation of new bone tissue [23, 24]. Current studies have shown that GDF5 can repair degenerative intervertebral discs and promote the proteoglycan and collagen type II protein expression $[25,26]$. Animal studies have found that the central region of intervertebral disc of GDF5 knockout mice showed low signal in MRI T2 weighted, the fibrous ring lost normal lamellar structure, NP atrophy and disorder, and the content of proteoglycan decreased significantly. The expression of proteoglycan and type II collagen mRNA decreased, which confirmed that the deletion of GDF5 gene was closely related to IVD degeneration. Meanwhile, GDF5 also promoted the differentiation of stem cells [27]. In this study, after GDF5 gene transfection of NPMSCs by lentivirus, mRNA and protein expression levels of KRT8, KRT18 and KRT19 in NPMSCs were significantly increased compared with other two words, suggesting that GDF5 gene can promote the differentiation of NPMSCs.

Regarding the mechanism of GDF5 acting on NPMSCs, Liu W found that GDF5 could inhibit the transcription and expression of RNA fragment microrna-34a, reduced the generate of IL-1 $\beta$, and increase the expression of proteoglycan and collagen II type. It indicates that GDF5 could delay or stop the degeneration of the disc [28]. Of course, the mechanism of GDF5 acting on NPMSCs still needs further study in follow-up experiments.

\section{Conclusion}

In this study, an in vitro culture model of NPMSCs was established, and gene transfection, qRT-PCR, WB and other techniques were used to prove the existence of NPMSCs in rabbit nucleus pulporeal tissue, GDF5 can promote the differentiation of rabbit NPMSCs into NPCs in vitro. The specific mechanism needs further study. Through the research of this subject, it is expected that the research results will be applied to the clinical application to solve the problem of inhibiting or reversing disc degeneration.

\section{Declarations}

\section{Ethics approval and consent to participate}

The experimental protocol was established, according to the ethical guidelines of the Helsinki Declaration and was approved by the Ethics Committee of The first affiliated hospital of bengbu medical college. Written informed consent was obtained from individual or guardian participants. 


\section{Consent for publication}

Written informed consent for publication was obtained from all participants.

\section{Availability of data and materials}

All data generated or analysed during this study are included in this published article.

\section{Competing interests}

The authors declare that they have no competing interests

\section{Funding}

Project of Natural Science Foundation of Anhui province(1908085MC90)

\section{Authors' contributions}

Gang Xu and Zhang Yao designed the experimental project. Yuchen Ye conducted literature review.Kun Zhu is responsible for laboratory implementation and paper writing. Zhao Rui did the article polishing and statistical analysis. All authors read and approved the final manuscript.

\section{Acknowledgements}

None

\section{References}

1. Kermani HR, Hoboubati H, Esmaeili-Mahani S, Asadi-Shekaari M. Induction of intervertebral disc cell apoptosis and degeneration by chronic unpredictable stress. J Neurosurg Spine. 2014;20:578-84.

2. Wang J, Chen H, Cao P, Wu X, Zang F, Shi L, Liang L, Yuan W. Inflammatory cytokines induce caveolin-1/ $\beta$-catenin signalling in rat nucleus pulposus cell apoptosis through the p38 MAPK pathway. Cell Prolif. 2016;49:362-72.

3. Han B, Wang HC, Li H, Tao YQ, Liang CZ, Li FC, Chen G, Chen QX. Nucleus pulposus mesenchymal stem cells in acidic conditions mimicking degenerative intervertebral discs give better performance than adipose tissue-derived mesenchymal stem cells. Cells Tissues Organs. 2015;199:342-52.

4. Mehrkens A, Matta A, Karim MZ, Kim S, Fehlings MG, Schaeren S, Erwin M. W. Notochordal cellderived conditioned medium protects human nucleus pulposus cells from stress-induced apoptosis. Spine J. 2017;17:579-88. 
5. Koppenaal T, Arensman RM, van Dongen JM, Ostelo R, Veenhof C, Kloek CJJ, Pisters MF. Effectiveness and cost-effectiveness of stratified blended physiotherapy in patients with non-specific low back pain: study protocol of a cluster randomized controlled trial. BMC Musculoskelet Disord. 2020;21:265.

6. Hu Y, Huang L, Shen M, Liu Y, Xiong L. Pioglitazone protects compression-mediated apoptosis in nucleus pulposus mesenchymal stem cells by suppressing oxidative stress. Oxid Med Cell Longev 2019; 2019: 1-14.

7. Karamini A, Bakopoulou A, Andreadis D, Gkiouras K, Kritis A. Therapeutic potential of mesenchymal stromal stem cells in rheumatoid arthritis: a systematic review of in vivo studies. Stem Cell Rev Rep. 2020;16:276-87.

8. Sheyn D, Ben-David S, Tawackoli W, Zhou Z, Salehi K, Bez M, De Mel S, Chan V, Roth J, Avalos P, Giaconi JC, Yameen H, Hazanov L, Seliktar D, Li D. Gazit D and Gazit Z. Human iPSCs can be differentiated into notochordal cells that reduce intervertebral disc degeneration in a porcine model. Theranostics. 2019;9:7506-24.

9. Nolta JA, Galipeau J, Phinney DG. Improving mesenchymal stem/stromal cell potency and survival: proceedings from the International Society of Cell Therapy (ISCT) MSC preconference held in May 2018, Palais des Congres de Montreal, Organized by the ISCT MSC Scientific Committee. Cytotherapy 2020; 22: 123-126.

10. Henry N, Clouet J, Le Bideau J, Le Visage C, Guicheux J. Innovative strategies for intervertebral disc regenerative medicine: From cell therapies to multiscale delivery systems. Biotechnol Adv. 2018;36:281-94.

11. Wang W, Deng G, Qiu Y, Huang X, Xi Y, Yu J, Yang X, Ye X. Transplantation of allogenic nucleus pulposus cells attenuates intervertebral disc degeneration by inhibiting apoptosis and increasing migration. Int J Mol Med. 2018;41:2553-64.

12. Qi L, Wang R, Shi Q, Yuan M, Jin M, Li D. Umbilical cord mesenchymal stem cell conditioned medium restored the expression of collagen II and aggrecan in nucleus pulposus mesenchymal stem cells exposed to high glucose. J Bone Miner Metab. 2019;37:455-66.

13. Li XC, Tang Y, Wu JH, Yang PS, Wang DL, Ruan DK. Characteristics and potentials of stem cells derived from human degenerated nucleus pulposus: potential for regeneration of the intervertebral disc. BMC Musculoskelet Disord. 2017;18:242.

14. Erwin WM, Islam D, Eftekarpour E, Inman RD, Karim MZ, Fehlings MG. Intervertebral disc-derived stem cells: implications for regenerative medicine and neural repair. Spine (Phila Pa 1976). 2013;38:2116.

15. Lin L, Jia Z, Zhao Y, Wu Y, Zhao X, Li Y, Guo Z, Chen J, Cheng S, Wang D, Ruan D. Use of limiting dilution method for isolation of nucleus pulposus mesenchymal stem/progenitor cells and effects of plating density on biological characteristics and plasticity. Biomed Res Int 2017; 2017: 9765843.

16. Zhang H, Ma X, Zhang L, Guan X, Bai T, Xue $C$. The ability to form cartilage of NPMSC and BMSC in SD rats. Int J Clin Exp Med. 2015;8:4989-96. 
17. Wang F, Gao ZX, Cai F, Sinkemani A, Xie ZY, Shi R, Wei JN, Wu XT. Formation, function, and exhaustion of notochordal cytoplasmic vacuoles within intervertebral disc: current understanding and speculation. Oncotarget. 2017;8:57800-12.

18. Rodrigues-Pinto R, Berry A, Piper-Hanley K, Hanley N, Richardson SM, Hoyland JA. Spatiotemporal analysis of putative notochordal cell markers reveals CD24 and keratins 8, 18, and 19 as notochordspecific markers during early human intervertebral disc development. J Orthop Res. 2016;34:132740.

19. Minogue BM, Richardson SM, Zeef LA, Freemont AJ, Hoyland JA. Transcriptional profiling of bovine intervertebral disc cells: implications for identification of normal and degenerate human intervertebral disc cell phenotypes. Arthritis Res Ther. 2010;12:R22.

20. Richardson SM, Ludwinski FE, Gnanalingham KK, Atkinson RA, Freemont AJ, Hoyland JA. Notochordal and nucleus pulposus marker expression is maintained by sub-populations of adult human nucleus pulposus cells through aging and degeneration. Sci Rep. 2017;7:1501.

21. Wang Z, Leng J, Zhao Y, Yu D, Xu F, Song Q, Qu Z, Zhuang X, Liu Y. N-cadherin maintains the healthy biology of nucleus pulposus cells under high-magnitude compression. Cell Physiol Biochem. 2017;43:2327-37.

22. Thorpe AA, Binch AL, Creemers LB, Sammon C, Le Maitre CL. Nucleus pulposus phenotypic markers to determine stem cell differentiation: fact or fiction? Oncotarget. 2016;7:2189-200.

23. Morimoto T, Kaito T, Matsuo Y, Sugiura T, Kashii M, Makino T, Iwasaki M, Yoshikawa H. The bone morphogenetic protein-2/7 heterodimer is a stronger inducer of bone regeneration than the individual homodimers in a rat spinal fusion model. Spine J. 2015;15:1379-90.

24. Itoh F, Watabe T, Miyazono K. Roles of TGF-beta family signals in the fate determination of pluripotent stem cells. Semin Cell Dev Biol. 2014;32:98-106.

25. Yan J, Yang S, Sun H, Guo D, Wu B, Ji F, Zhou D. Effects of releasing recombinant human growth and differentiation factor- 5 from poly (lactic-co-glycolic acid) microspheres for repair of the rat degenerated intervertebral disc. J Biomater Appl. 2014;29:72-80.

26. Clarke LE, McConnell JC, Sherratt MJ, Derby B, Richardson SM, Hoyland JA. Growth differentiation factor 6 and transforming growth factor-beta differentially mediate mesenchymal stem cell differentiation, composition, and micromechanical properties of nucleus pulposus constructs. Arthritis Res Ther. 2014;16:R67.

27. Feng $\mathrm{C}$, Liu H, Yang $\mathrm{Y}$, Huang B, Zhou Y. Growth and differentiation factor-5 contributes to the structural and functional maintenance of the intervertebral disc. Cell Physiol Biochem. 2015;35:116.

28. Liu W, Zhang Y, Feng X, Li S, Gao Y, Wang K, Song Y, Yang S, Tu J, Shao Z, Yang C. Inhibition of microRNA-34a prevents IL-1 beta-induced extracellular matrix degradation in nucleus pulposus by increasing GDF5 expression. Exp Biol Med (Maywood). 2016;241:1924-32.

\section{Figures}


A

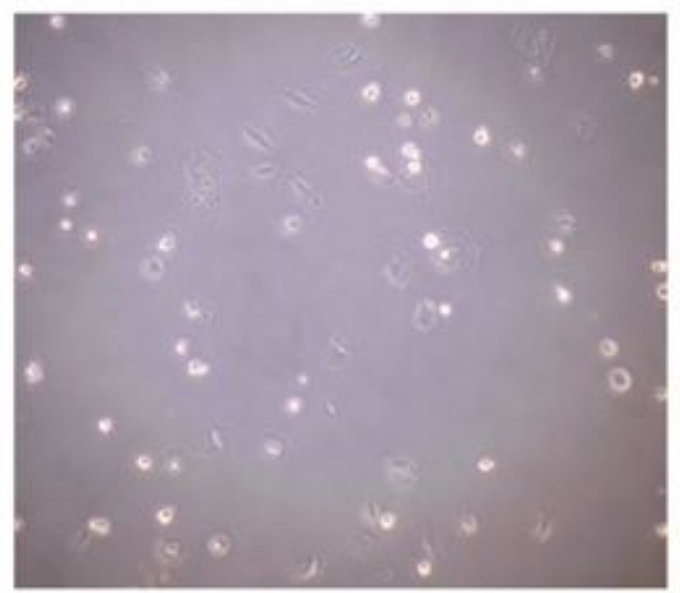

C

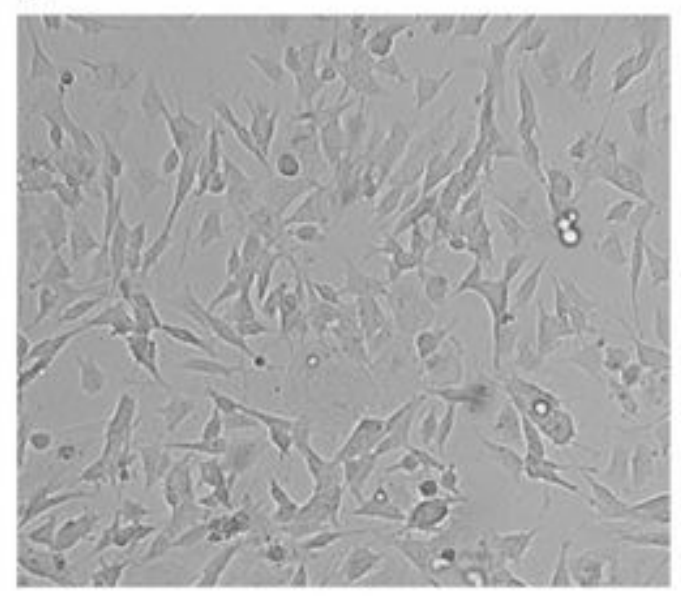

B

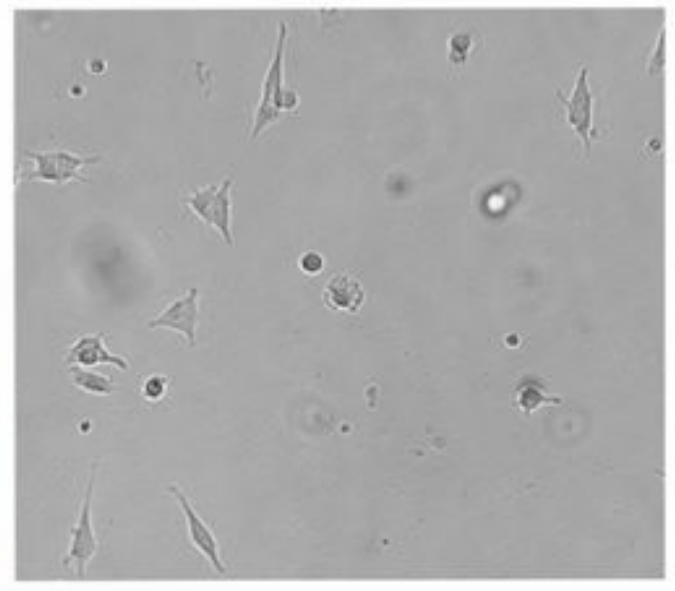

D

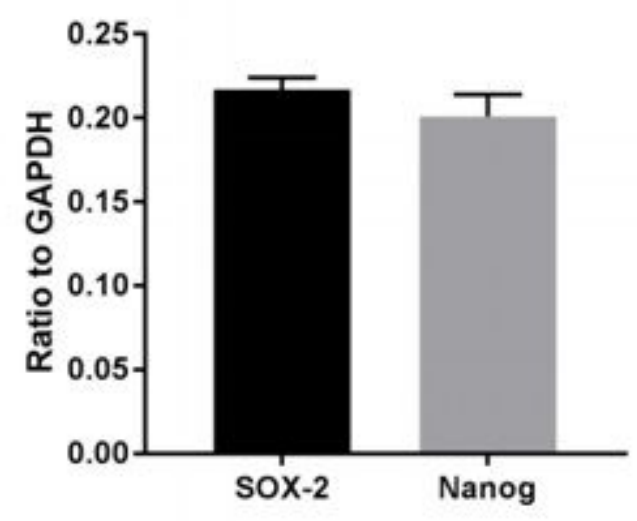

\section{Figure 1}

Primary, passage and transfected NPMSCs. A. Completely digested NPMSCs (magnification, $\times 200$ ); $B$. Primary NPMSCs (magnification, $\times 200$ ); C. Third generation (magnification, $\times 200$ ); D. qRT-PCR results: NPMSCs could express stem cell gene SOX2, Nanog. 
A

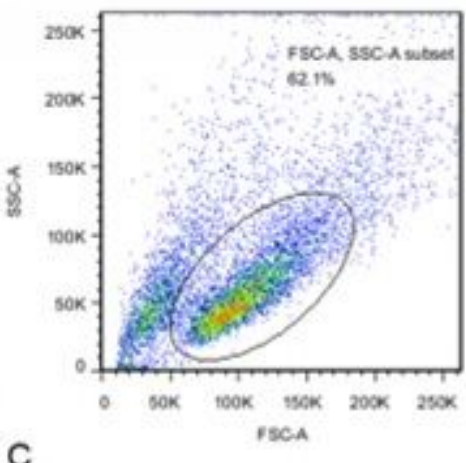

C

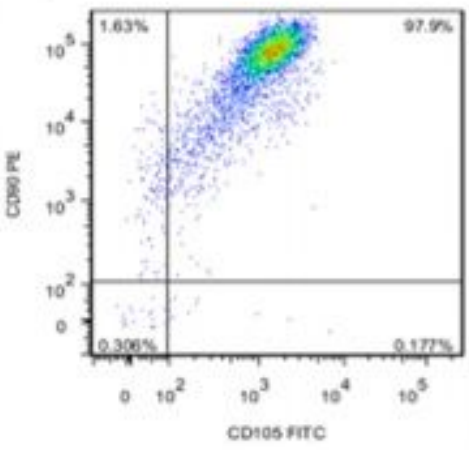

B

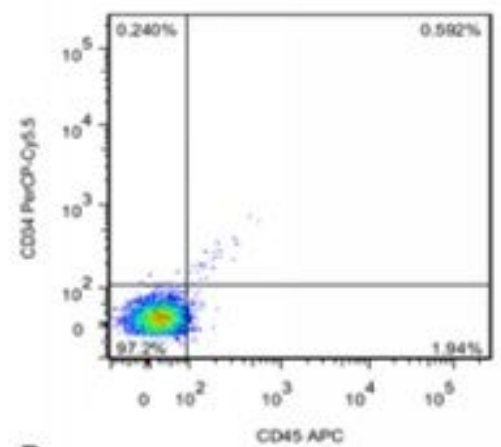

D

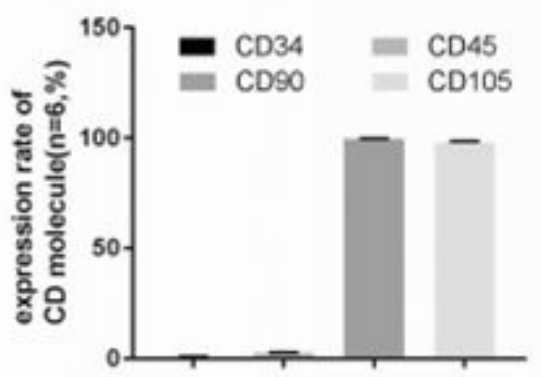

\section{Figure 2}

Identification of NPMSCs by FCM. A. Remove cell fragments by FSC/SSC scatter plot, and circle NPMSCs. B. The immunophenotypes CD34 and CD45 of hematopoietic stem cells were negative. C. The immunophenotypes CD90 and CD105 expressed positive. D. The expression rate of CD molecule of surface immunophenotype of NPMSCs.

A

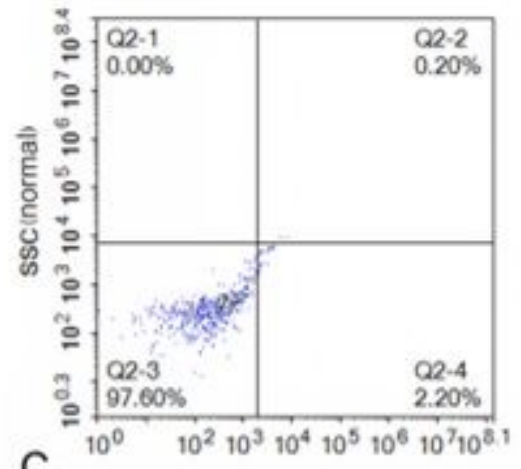

C

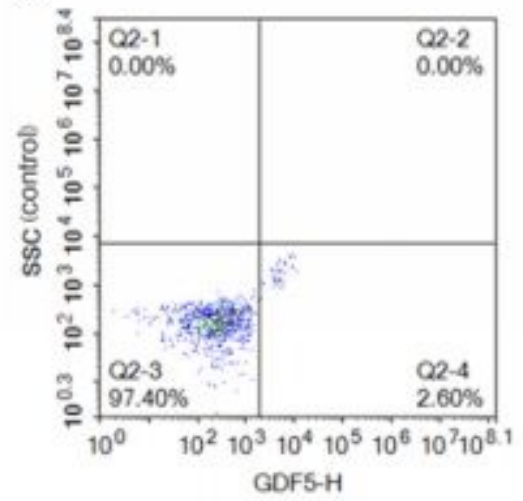

B
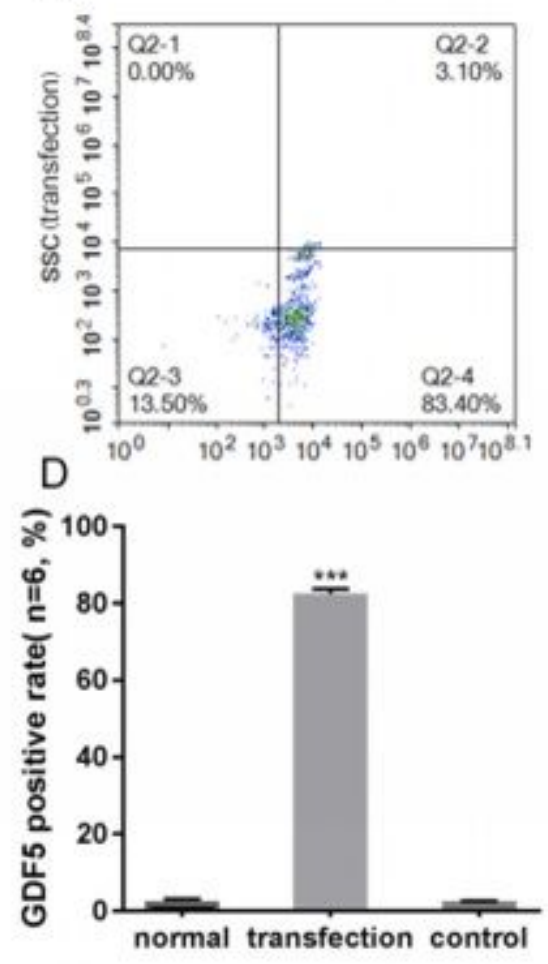


\section{Figure 3}

FCM cell transfection rate.A.GDF5 positive rate in normal group; B.GDF5 positive rate (\%) in the transfection group; C.GDF5 positive rate of control group; D.The GDF5 positive rate of the transfection group was significantly higher than that of the normal group and the control group, and the difference was statistically significant $(P<0.001)$.

A

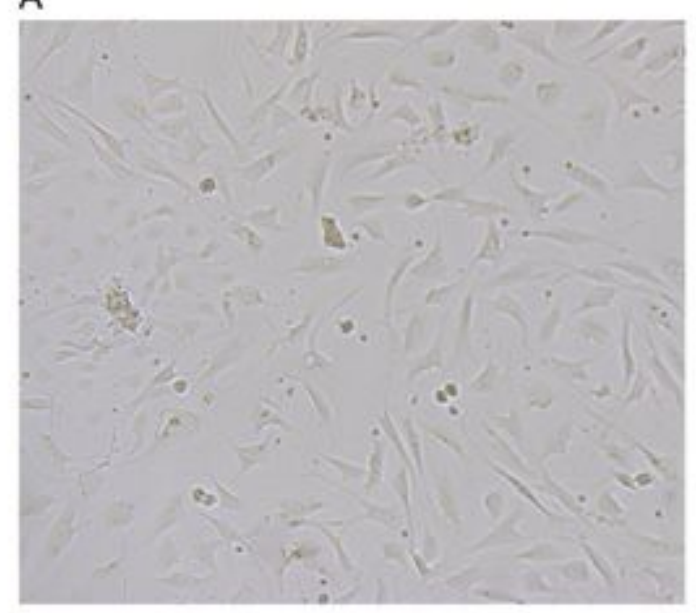

C

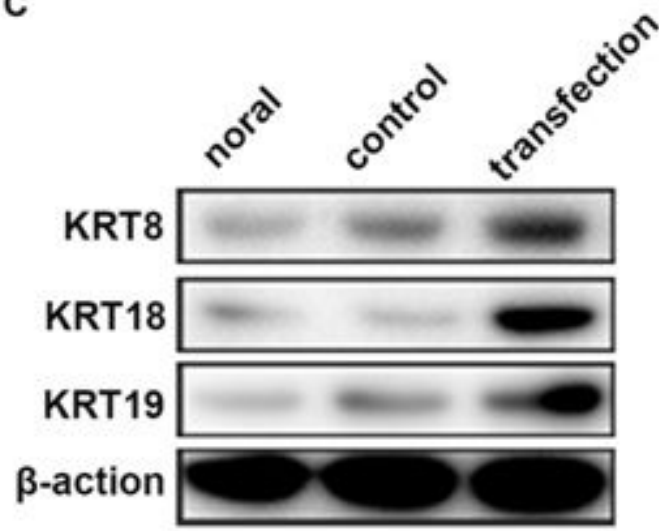

B

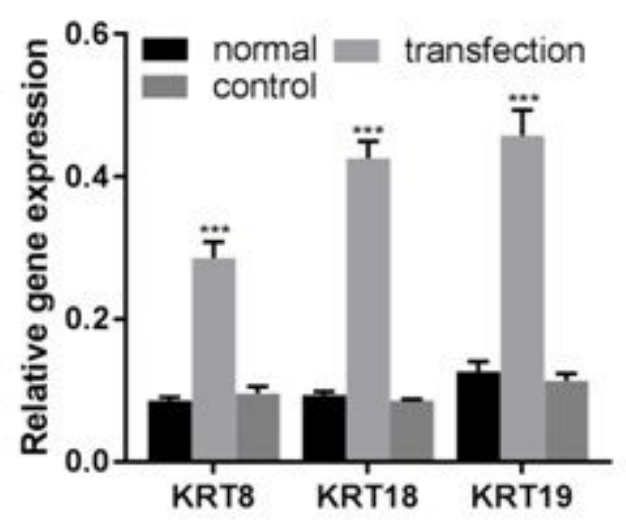

D

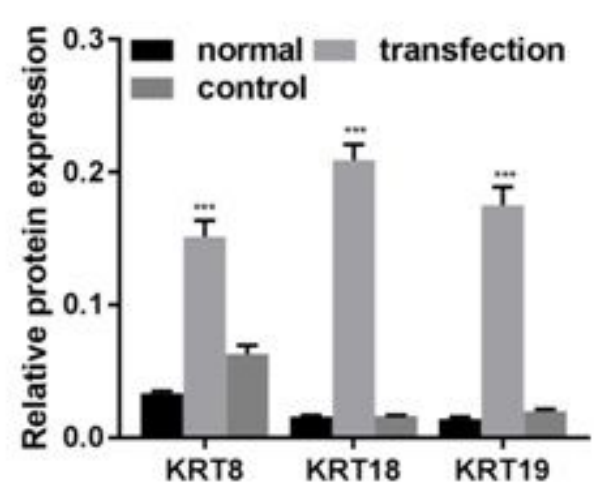

\section{Figure 4}

A. Recombinant NPMSCs after transfection(magnification, $\times 200$ ); B.qRT-PCR results: mRNA expression levels of KRT8, KRT18 and KRT19 were significantly increased in the transfection group compared with the other two groups $(P<0.001)$. C. WB detected the protein expression levels of KRT8, KRT19 and KRT19 in the three groups. D. WB results:protein expression levels of KRT8, KRT18 and KRT19 were significantly increased in the transfection group compared with the other two groups $(P<0.001)$. 